\title{
In memory of Bruce McEwen: a gentle giant of neuroscience
}

\author{
On 2 January 2020, the neuroscience community lost not only a pioneering figure, but also a generous and \\ influential thought leader. Bruce Sherman McEwen, head of the Harold and Margaret Milliken Hatch Laboratory \\ of Neuroendocrinology at the Rockefeller University, passed away at age 81, following a short illness. A member \\ of the National Academy of Sciences, National Academy of Medicine and American Academy of Arts \& Sciences, \\ and former president of the Society for Neuroscience, Bruce will be remembered for his profound scientific impact, \\ measured not only by output of papers, but also by the large family of neuroscientists he trained over a career \\ spanning nearly six decades. Above all, Bruce will be remembered for his generosity, kindness, gentleness of soul, \\ and for being an extraordinary mentor.
}

B orn 17 January 1938, Bruce obtained a Bachelor's of Science in chemistry from Oberlin College, followed by a PhD in cell biology from Rockefeller University in 1964. Following a short time in Sweden furthering his training, Bruce returned to the US and founded the Laboratory of Neuroendocrinology in 1966. From his first paper in 1959 to the time of his death, Bruce published over 1,000 scientific articles (with over 130,000 citations) on topics ranging from molecular mechanisms of steroid action in the brain to the health impacts of early-life adversity. The breadth of his work and interests led Bruce to describe himself later in his career as a "molecular sociologist."

While the effect of stress hormones on the brain is taken for granted now, it was Bruce's 1968 paper in Nature that first demonstrated receptors for the adrenal stress steroid, corticosterone, in the hippocampus. This seminal finding led to recognition that steroid hormones have a direct impact on neural function. Over the subsequent five decades, Bruce continued to lead the field in understanding the neurobiology of stress. Work by his lab, and by his myriad of trainees, revealed neural plasticity influenced by stressful experience and created a template for understanding how these alterations could influence behavior, from affect to cognition. His work showing stress-induced structural plasticity of dendrites and regulation of adult neurogenesis in the hippocampus revealed mechanisms by which stress exposure remodels corticolimbic circuits and influences susceptibility to psychiatric illnesses. Bruce also studied how stress at different life stages could have different effects, with early-life stress leaving a lasting impact on the developing brain, while aging increased vulnerability to stress, contributing to accelerated cognitive

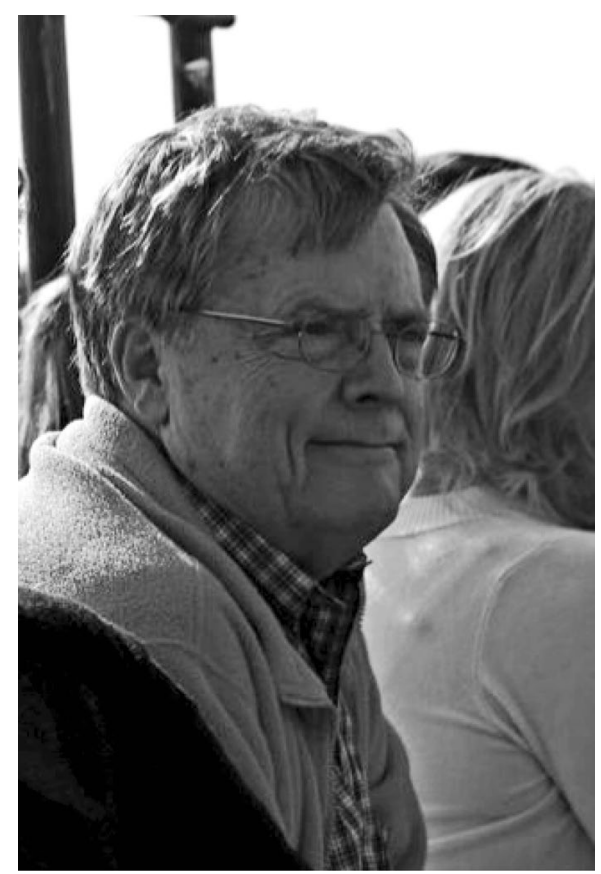

Credit: Tiffany Lee

decline. Work from Bruce's lab also set the stage for how we now understand stress to be a contributing factor in the development of chronic inflammatory and metabolic diseases. This aspect of Bruce's work highlighted reciprocal relationships between the brain and body as being critical to both susceptibility and resilience to stress.

Bruce was a key figure in developing the concept of 'allostatic load', which posits that physiological adaptation to persistent or repeated challenges imparts a cost in the form of cumulative wear and tear on the body. This conceptualization of the health impacts of stress had an unquestionable impact on the field of stress biology and still represents the primary theoretical model by which we understand how adverse life events impact physical and mental health.

In addition to his work on stress, Bruce was a pioneer in understanding how gonadal steroids act in the brain. His early work helped to map brain estrogen receptors, and his lab discovered that reproductive hormones could influence structural plasticity of dendrites and synapses. Bruce's work in this area contributed to the understanding of sex differences and was foundational for the now-widespread recognition of the need to consider sex as a biological variable in neuroscience and beyond.

In both his personal and professional life, Bruce was a family man. He was an adoring husband, father and grandfather. Bruce's scientific family came to lovingly refer to each other as the 'McEwenites', brought together across generations and research fields by Bruce's generous mentorship. Bruce frequently adopted neuroscientists from outside his lab into this scientific family, treating all to his scientific and personal generosity. Without regard for his own professional stature, Bruce never viewed his trainees as competition, instead supporting everyone to take their ideas and flourish and go on to do great things independently. It is rare to find an individual who can claim such a long and wide lineage of successful scientific offspring.

While this obituary might attempt to spell out a small fraction of his achievements, Bruce rarely, if ever, discussed his own accomplishments, legacy or impact in science. Bruce did what he did for the generation of knowledge and the advancement of society, not for accolades. He had an infectious energy and optimism. Whether it came in an hourslong discussion about a finding in the lab or a comprehensive review article, Bruce appreciated any advance and celebrated 
every success. He was patient and generous and gave his time and energy to everyone and anyone. One was just as likely to see him talking to an undergraduate student at their poster as to see him rubbing shoulders with Nobel laureates or celebrities. In his actions, Bruce reminded us that, ultimately, we are all the same and it is by working together that we can achieve great things in science.

Bruce McEwen's passing will have a resounding impact on the neuroscience community and will not be easy to recover from. His legacy will be carried on by the hundreds of McEwenites and the thousands more influenced by his work who will continue to advance knowledge and scientific discovery. Bruce's goal was to bring humanity into science and use knowledge, and compassion, to improve the human condition. We, as authors, are a few of the fortunate group of scientists who had the privilege to have trained with and learned from Bruce and to be directly influenced by his intellect, generosity and optimism. However, we all, scientists and non-scientists alike, would benefit from adopting the gentle demeanor, modesty and generosity that Bruce exhibited to everyone he met.

Matthew N. Hill1 ${ }^{1 凶}$, Ilia N. Karatsoreos (D) 2 , E. Ron de Kloet ${ }^{3}$, Sonia Lupien ${ }^{4}$ and Catherine S. Woolley ${ }^{5}$
${ }^{1}$ Departments of Cell Biology and Anatomy, Mathison Centre for Mental Health Research and Education, Hotchkiss Brain Institute, University of Calgary, Calgary, Alberta, Canada. ${ }^{2}$ Department of Psychological and Brain Sciences and Center for Neuroendocrine Studies, University of Massachusetts Amherst, Amherst, MA, USA. ${ }^{3}$ Department of Endocrinology, Leiden University Medical Center, Leiden, The Netherlands. ${ }^{4}$ Centre for Studies on Human Stress, Institut Universitaire en Santé Mentale de Montréal, Montreal, Québec, Canada. ${ }^{5}$ Department of Neurobiology, Northwestern $\neg$ University, Evanston, IL, USA.

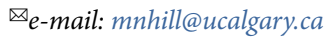

Published online: 23 March 2020 https://doi.org/10.1038/s41593-020-0613-y 